\title{
CDISC SDTM Tumor Response Assessment Test Name Terminology
}

National Cancer Institute

\section{Source}

National Cancer Institute. CDISC SDTM Tumor Response Assessment Test Name

Terminology. NCI Thesaurus. Code C96781.

Terminology associated with the tumor response assessment test name codelist of the Clinical Data Interchange Standards Consortium (CDISC) Study Data Tabulation Model (SDTM). 